\title{
Antimelanogenic Efficacy of Melasolv (3,4,5-Trimethoxycinnamate Thymol Ester) in Melanocytes and Three-Dimensional Human Skin Equivalent
}

\author{
John Hwan Lee ${ }^{a, e}$ Eun-Soo Lee ${ }^{a} \quad$ Il-Hong Bae ${ }^{a, d}$ Jeong-Ah Hwang ${ }^{a}$ \\ Se-Hwa Kim ${ }^{\text {b, c }}$ Dae-Yong Kim ${ }^{d}$ Nok-Hyun Park ${ }^{a}$ Ho Sik Rho ${ }^{a}$ Yong Jin Kim ${ }^{a}$ \\ Seong-Geun Oh ${ }^{\mathrm{e}}$ Chang Seok Lee ${ }^{\mathrm{a}}$ \\ ${ }^{a}$ Amorepacific Corporation R\&D Center, Yongin, ${ }^{b}$ Department of Medical Physics, University of Science and \\ Technology, and ' Center for Nano-Bio Measurement, Korea Research Institute of Standards and Science, Daejeon, \\ ${ }^{d}$ College of Veterinary Medicine, Seoul National University, and ${ }^{e}$ Department of Chemical Engineering, \\ Hanyang University, Seoul, Republic of Korea
}

\section{Keywords}

Melasolv · Melanogenesis · Depigmentary agent ·

Three-dimensional human skin equivalent

\begin{abstract}
Background/Aims: Excessive melanogenesis often causes unaesthetic hyperpigmentation. In a previous report, our group introduced a newly synthesized depigmentary agent, Melasolv ${ }^{\mathrm{TM}}$ (3,4,5-trimethoxycinnamate thymol ester). In this study, we demonstrated the significant whitening efficacy of Melasolv using various melanocytes and human skin equivalents as in vitro experimental systems. Methods: The depigmentary effect of Melasolv was tested in melan-a cells (immortalized normal murine melanocytes), a-melanocytestimulating hormone (a-MSH)-stimulated B16 murine melanoma cells, primary normal human melanocytes (NHMs),
\end{abstract}

John Hwan Lee and Eun-Soo Lee contributed equally to this work. Seong-Geun Oh and Chang Seok Lee are co-corresponding authors.

\section{KARGER}

(c) 2017 S. Karger AG, Basel

E-Mail karger@karger.com

www.karger.com/spp and human skin equivalent (MelanoDerm). The whitening efficacy of Melasolv was further demonstrated by photography, time-lapse microscopy, Fontana-Masson (F\&M) staining, and 2-photon microscopy. Results: Melasolv significantly inhibited melanogenesis in the melan-a and a-MSHstimulated B16 cells. In human systems, Melasolv also clearly showed a whitening effect in NHMs and human skin equivalent, reflecting a decrease in melanin content. F\&M staining and 2-photon microscopy revealed that Melasolv suppressed melanin transfer into multiple epidermal layers from melanocytes as well as melanin synthesis in human skin equivalent. Conclusion: Our study showed that Melasolv clearly exerts a whitening effect on various melanocytes and human skin equivalent. These results suggest the possibility that Melasolv can be used as a depigmentary agent to treat pigmentary disorders as well as an active ingredient in cosmetics to increase whitening efficacy.

(c) 2017 S. Karger AG, Basel
Chang Seok Lee, $\mathrm{PhD}$

Amorepacific Corporation R\&D Center

1920 Yonggu-daero, Giheung-gu

Yongin, Gyeonggi-do, 17074 (Republic of Korea)

E-Mail cslee2010@ amorepacific.com

Seong-Geun $\mathrm{Oh}, \mathrm{PhD}$

Department of Chemical Engineering, Hanyang University Wangsimni-ro, Seongdong-gu, Seoul, 04763 (Republic of Korea)

E-Mail seongoh@ hanyang.ac.kr 


\section{Introduction}

Melanogenesis is the process of melanin production that serves to protect skin against deleterious ultraviolet (UV) irradiation [1]. However, overexpression of melanin causes freckles, senile lentigines, and other forms of melanin hyperpigmentation, which are serious human aesthetic problems [2]. However, despite the development of numerous depigmentary agents, none has proved clinically satisfactory in terms of safety or efficacy.

Tyrosinase, the rate-limiting enzyme of the melanogenic pathway, is a copper-containing glycoprotein of approximately $60-70 \mathrm{kDa}$ and a common target for therapeutic agents intended to alleviate cutaneous hyperpigmentation [3]. Despite the large number of compounds that inhibit tyrosinase in vitro, only a few have been shown able to induce real effects in clinical trials. The gap between in vitro and in vivo studies suggests that innovative strategies are needed to validate the efficacy and safety of such agents [4].

To obtain effective and safe topical depigmenting agents, we previously synthesized and screened several compounds for high-level inhibitory activity against melanin synthesis using the normal murine melanocyte cell line, melan-a. Ultimately, we discovered that 3,4,5-trimethoxycinnamate thymol ester (TCTE), synthesized by conjugating 3,4,5-trimethoxycinnmic acid with thymol, showed potent depigmenting effects and low cytotoxicity [5]. We named this compound "Melasolv", a trademark of the Amorepacific Corporation for this depigmentary ingredient.

However, although it is known that Melasolv ${ }^{\mathrm{TM}}$ inhibits melanin synthesis in melan-a cells, there has been a growing need to demonstrate that it exerts similar effects on a human system as well as in other cell lines. Thus, to more effectively evaluate the depigmentary efficacy of Melasolv, we used various additional experimental systems, namely an $\alpha$-melanocyte-stimulating hormone (a-MSH)-stimulated B16 murine melanoma cell line, primary normal human melanocytes (NHMs), and a human skin equivalent system (MelanoDerm). We then analyzed the results using photography, time-lapse microscopy, tissue staining, and 2-photon microscopy. Collectively, our results demonstrated that Melasolv exhibited whitening efficacy regardless of cell type or test condition.

\section{Materials and Methods}

\section{Chemicals}

Melasolv was synthesized by our research group, as described previously [5]. Kojic acid and a-MSH were purchased from SigmaAldrich (St. Louis, MO, USA).

Melasolv Inhibits Melanogenesis

\section{Cell Culture}

Melan-a cells were maintained in monolayers at $37^{\circ} \mathrm{C}$ in a humidified $10 \% \mathrm{CO}_{2}$ incubator in RPMI-1640 medium (Lonza, Basel, Switzerland) containing 10\% FBS (Gibco, NY, USA); 100 U/ml potassium penicillin and $100 \mathrm{mg} / \mathrm{mL}$ streptomycin sulfate (Lonza); and phorbol 12-myristate 13-acetate (Sigma-Aldrich). B16 murine melanoma cells (American Type Culture Collection [ATCC]; Manassas, VA, USA) were cultured in DMEM containing $10 \% \mathrm{FBS}$ (ATCC) and incubated at $37^{\circ} \mathrm{C}$ in a humidified $5 \%$ $\mathrm{CO}_{2}$ atmosphere. Primary NHMs from the neonatal foreskin of a darkly pigmented donor were purchased from Cascade Biologics (\#C2025C; Portland, OR, USA) and cultured in Medium 254 (\#M254-500; Cascade Biologics) supplemented with Human Melanocyte Growth Supplement (HMGS-2, S-016-5; Cascade Biologics). Primary cells for experiments were used between passage 4 and 7 .

\section{Cell Viability Assay}

Melan-a cells were seeded onto 96 -well plates $\left(1 \times 10^{4}\right.$ cells/ well) and cultured with Melasolv for $72 \mathrm{~h}$, after which an MTT solution was added and the cells were incubated for $30 \mathrm{~min}$. The dark blue formazan crystals that formed in intact cells were dissolved in DMSO, and absorbance was measured at $570 \mathrm{~nm}$ using a microplate reader.

\section{Measurement of Melanin Content}

Melan-a cells and NHMs were treated with the indicated concentrations of Melasolv without additional stimuli. B16 cells were treated with the indicated concentrations of Melasolv, and then incubated in culture media containing $\alpha$-MSH $(200 \mathrm{nM})$ for 3 days. Thereafter, cells were washed twice with PBS, and lysed by incubating in $200 \mu \mathrm{L}$ of $1 \mathrm{~N} \mathrm{NaOH}$ at $60^{\circ} \mathrm{C}$ for $1 \mathrm{~h}$. Cell lysates were transferred to a 96-well plate, and absorbance was measured at $475 \mathrm{~nm}$. The total protein content in each experimental group was then measured using a DC protein assay (BioRad, Hercules, CA, USA), and the calculated melanin content in each experimental group was expressed relative to total protein.

\section{Time-Lapse Melanin Imaging}

For melanin assays, B16 cells were cultured in a 12-well plate for $24 \mathrm{~h}$ at $37^{\circ} \mathrm{C}$ in a $10 \% \mathrm{CO}_{2}$ incubator. Then, after treatment of each group with $\alpha$-MSH and Melasolv, bright-field images of live cell cultures in a $10 \% \mathrm{CO}_{2}$ incubator were recorded every hour for $70 \mathrm{~h}$ using a JuLI Stage real-time cell history recorder (NanoEnTek, South Korea) connected to a JuLI Stage microscope equipped with a $\times 20$ objective lens. Three different regions per group were randomly selected and analyzed. Dark dot signals in B16 cells in the bright-field images indicate intracellular melanin. The LED light conditions used in this study caused no phototoxicity (data not shown).

\section{Three-Dimensional Human Skin Equivalent}

As a human skin tissue model, we used MelanoDerm (MEL300-B; MatTek Corp., Ashland, MA, USA), a viable, reconstituted, 3-dimensional (3D) human skin equivalent derived from black donors, which contains normal melanocytes and keratinocytes. MelanoDerm was maintained in EPI-100-NMM-113 medium as recommended by the manufacturer. Melasolv $(0.1 \%)$ was applied to MelanoDerm on days 1, 4, 6, 8, 11, 13, and 15. MelanoDerm can be treated with test compound up to 21 days, according to the experimental protocol of MatTek Corp. Prior to Melasolv treatment, tissues were washed with $1 \mathrm{~mL}$ of PBS to remove any residual com-

Skin Pharmacol Physiol 2017;30:190-196

DOI: $10.1159 / 000477356$ 


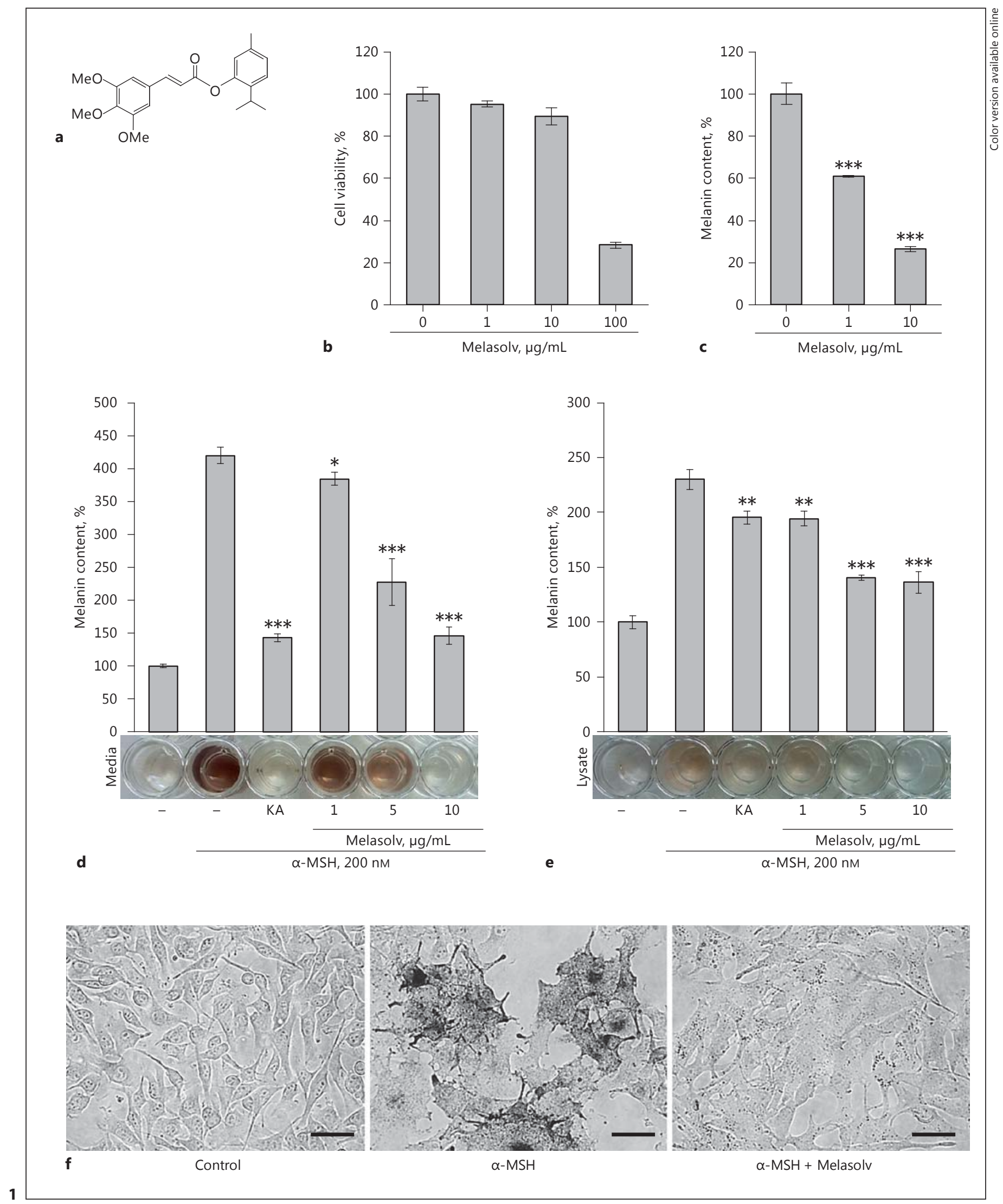

(For legend see next page.) 
pound. After 18 days, MelanoDerm tissues were fixed in $4 \%$ buffered formaldehyde, embedded in paraffin, cut to a thickness of $3 \mu \mathrm{m}$, and stained with F\&M staining solution.

Two-Photon Excitation Fluorescence Imaging

In this study, we used an $f s$-pulsed Ti:sapphire laser (Chameleon Vision-S, Coherent Inc., Santa Clara, CA, USA) capable of tuning wavelengths from 690 to $1,050 \mathrm{~nm}$, with a pulse width of 75 $f s$ and a repetition rate of $80 \mathrm{MHz}$ [6]. The laser beam was delivered into an inverted microscope (FV1000MPE +IX81; Olympus, Tokyo, Japan). For imaging melanin in the MelanoDerm model, we used an excitation wavelength of $800 \mathrm{~nm}$, and collected emitted signals at a wavelength of 490 to $540 \mathrm{~nm}$ using a bandpass filter [7]. A $\times 60$ water-immersion UPlanSApo/UIS2 objective lens (numerical aperture [NA] 1.2; Olympus) or a 30 silicon-immersion objective lens (NA 1.05) was used to focus the laser beam into the sample. For sample preparation, each MelanoDerm preparation was fixed by incubating with a $4 \%$ formalin solution for $24 \mathrm{~h}$ at $4^{\circ} \mathrm{C}$. After washing with a PBS/ $0.1 \%$ BSA (bovine serum albumin) solution, the basement membrane sides and stratum corneum sides of the MelanoDerm sample were imaged separately to measure intracellular melanin in the melanocyte layer and extracellular melanin in the stratum corneum layer, respectively. Measurement volume was $200(x) \times 200(y) \times 100(z) \mu \mathrm{m}^{3}$, and 51 image slices $(z$-depth interval $=2 \mu \mathrm{m}$ ) were reconstructed to produce each 3D image.

Statistical Analysis

Data are expressed as means \pm SD (standard deviation) values, and the Student $t$ test was used for statistical comparisons. A $p$ value $<0.05$ was considered statistically significant (see figure legends for individual $p$ values).

\section{Results and Discussion}

Prior to examining the efficacy of Melasolv, we synthesized Melasolv (Fig. 1a) as described in our previous report [5]. This previous study showed that Melasolv exhibited the most potent depigmenting effect $\left(\mathrm{IC}_{50} \approx 3.7 \mu \mathrm{g} /\right.$ $\mathrm{mL}$ ) of all compounds tested in screening assays using melan-a cells, and showed low cytotoxicity $\left(\mathrm{CC}_{50}>74 \mu \mathrm{g} /\right.$ $\mathrm{mL}$ ). In this study, we have reconfirmed the whitening ability of Melasolv at different concentrations in melan-a cells. Melasolv caused no apparent cytotoxicity at concentrations up to $10 \mu \mathrm{g} / \mathrm{mL}$ and also inhibited melanin synthesis (Fig. 1b, c), as previously reported. Melanin as-

Fig. 1. The depigmentary efficacy of Melasolv in melan-a and B16 murine cell line. a Chemical structure of Melasolv. b Effects of Melasolv on the viability of melan-a cells. Cells were treated with the indicated concentrations of Melasolv for $72 \mathrm{~h}$. Values are means \pm SD of 3 determinations, expressed as a percentage of the control. c Effects of Melasolv on melanin synthesis in melan-a cells. Values are means \pm SD of 3 determinations expressed as a percentage of the control. Effects of Melasolv on secreted (extracellular) melanin (d) and intracellular melanin (e) contents in says using a-MSH-stimulated mouse B16 melanoma cells are a representative and sensitive in vitro model for detecting changes in the level of intracellular and extracellular melanin content induced by putative bioactive compounds [8]. Thus, we treated $\alpha-\mathrm{MSH}$-stimulated B16 cells with Melasolv and determined the extracellular and intracellular melanin content. Melasolv significantly inhibited melanin secretion into the culture media in a concentration-dependent manner (Fig. 1d), and clearly decreased the amount of synthesized melanin detected in whole-cell lysates (Fig. 1e). This latter effect was confirmed in photographs of treated cells (Fig. 1f). To observe the antimelanogenic effect of Melasolv over time, we monitored $\alpha$-MSH-stimulated B16 cells using timelapse microscopy. As shown in the online supplementary movie (see www.karger.com/doi/10.1159/000477356 for all online suppl. material), Melasolv retarded the melanin synthesis induced by a-MSH stimulation in B16 cells, measured after allowing a sufficient time for melanogenesis to occur. These results indicate that Melasolv suppresses melanogenesis in murine melanocyte cell lines, such as B16 cells and melan-a cells, despite a robust melanogenic stimulus.

Next, we sought to determine whether Melasolv also exerted a depigmenting effect on human cells. To this end, we first treated NHMs, darkly pigmented neonatal melanocytes, with Melasolv for 7 days. Melasolv caused no apparent cytotoxicity at concentrations up to $10 \mu \mathrm{g} / \mathrm{mL}$ and suppressed melanin synthesis in a concentration-dependent manner (Fig. 2a, b), producing effects similar to those observed in murine cells. To extend this investigation to a more physiological setting, we tested the whitening ability of Melasolv in a 3D human skin equivalent, which closely resembles human skin in architecture and composition, with all major cell types represented in physiologically relevant ratios [9]. For this, we used MelanoDerm (MEL300-B), which features a stratified, differentiated epidermal layer consisting of keratinocytes and melanocytes. As described in the Materials and Methods section, Melasolv $(0.1 \%)$ was topically applied to MelanoDerm samples for 18 days, after which, changes in the color of tissue and

a-MSH-stimulated B16 cells. B16 cells were treated with the indicated concentrations of Melasolv and $\alpha-\mathrm{MSH}$ for 3 days. Then, the lysates were estimated by absorbance at $405 \mathrm{~nm}$. The melanin contents were normalized for total protein contents. Kojic acid (KA; $50 \mu \mathrm{g} / \mathrm{mL}$ ) is used as a reference compound, which is a representative antimelanogenic agent [4]. f Bright-field images of B16 melanoma cell line treated with $\alpha-\mathrm{MSH}(200 \mathrm{nM}) \pm$ Melasolv $(10 \mu \mathrm{g} / \mathrm{mL})$ for $72 \mathrm{~h}$. Dark dot-like patterns indicate intracellular melanosome. Scale bar, $50 \mu \mathrm{m} .{ }^{*} p<0.05$; $^{* *} p<0.01$; $^{* *} p<0.001$.

Skin Pharmacol Physiol 2017;30:190-196 DOI: $10.1159 / 000477356$ 


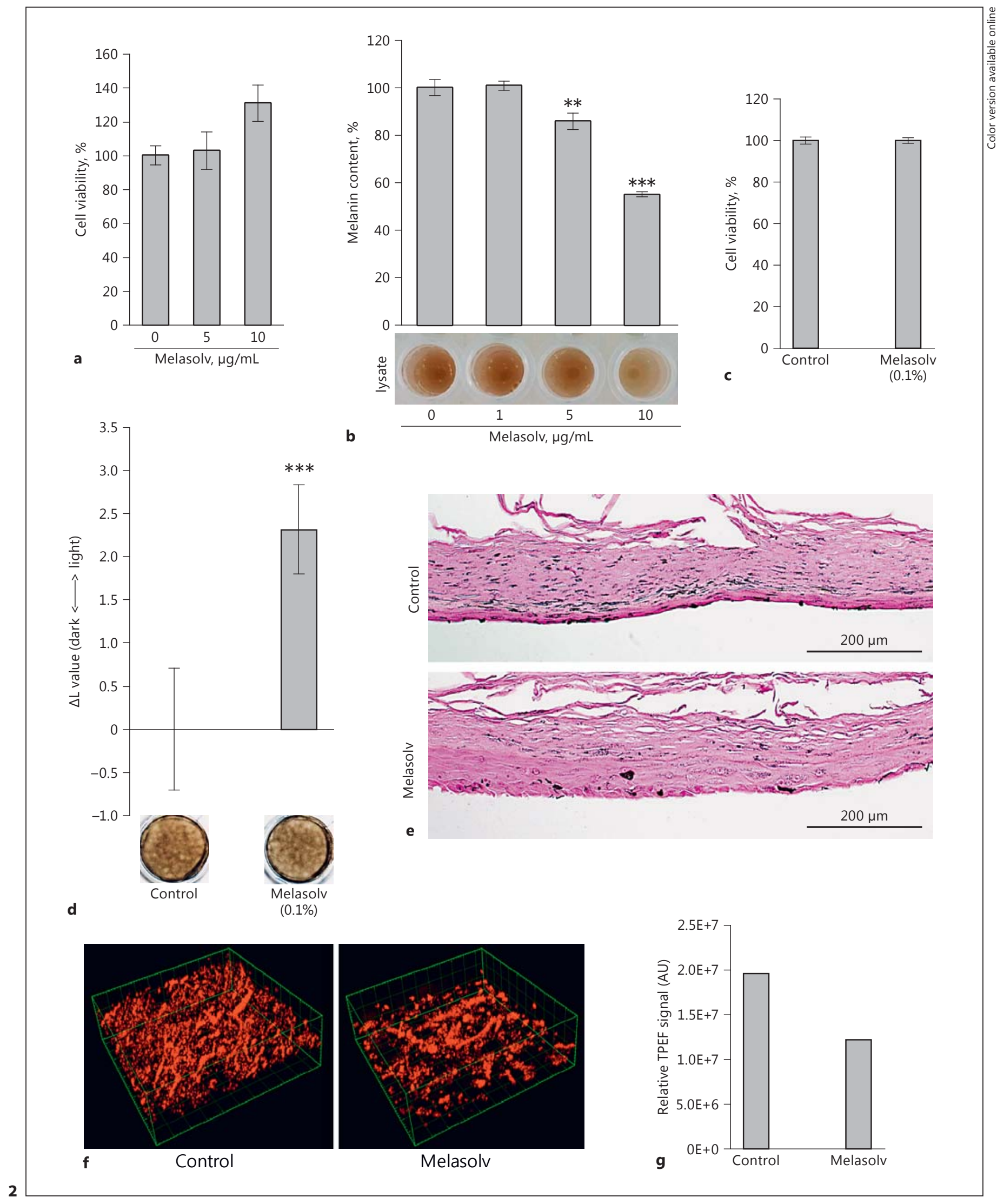

(For legend see next page.) 
melanin content were assessed. Melasolv-treated tissue caused no apparent cytotoxicity and appeared lighter than control tissue after 18 days (Fig. 2c), as demonstrated by photography and measurement of the L-values, a lightness/darkness index (Fig. 2d). Moreover, F\&M staining of tissue sections revealed that Melasolv decreased the melanin content in the whole tissue as well as the number of hyperpigmented melanocytes in the basal layer (Fig. 2e). The poor morphology of the tissue section might have been due to a prolonged culture of the models necessary for the effect of the novel antimelanogenic substance. To further observe changes in melanin content in detail, we performed 2-photon excitation fluorescence (TPEF) imaging experiments. In $3 \mathrm{D}$ human skin equivalent, melanocytes are localized at the basement membrane, where they are interspersed with basal layer keratinocytes. Thus, we compared the autofluorescence signals of melanin in the melanocyte layer of control and Melasolv-treated groups using TPEF microscopy (Fig. 2f). An analysis of these images showed that the TPEF signal intensity for melanin in the melanocyte-rich area was decreased by approximately 38\% in Melasolv-treated tissues compared with control tissues (Fig. 2g). On the basis of these data, we conclude that Melasolv clearly exerts a whitening ability through inhibition of melanin synthesis in hyperpigmented human melanocytes and 3D human skin equivalent, similar to the effects observed in murine melanocyte cell lines.

Although we selected Melasolv as the best depigmenting agent among the synthetic compounds in our previous report, the antimelanogenic efficacy of the compounds was screened only in the melan-a murine melanocyte cell line. Because it is conceivable that an active compound might function differently depending on species or cell line [10], we have confirmed the whitening activity of Melasolv in other cell lines and species in this study. As shown above, we clearly demonstrated that Melasolv possessed antimelanogenic activity independent of melanocyte cell lines and species. In addition, we sought to demonstrate the inhibitory effect of Melasolv on melanin synthesis using various in vitro experimental tools. In particular, the experimental

Fig. 2. Effects of Melasolv on NHMs and human skin equivalents. a Effects of Melasolv on the viability of NHMs. b NHMs were treated with the indicated concentrations of Melasolv for 7 days. The lysates were estimated by absorbance at $405 \mathrm{~nm}$. The melanin contents were normalized for total protein contents. c Viability test of $0.1 \%$ Melasolv treatment on human skin equivalents. d Human skin equivalents (MelanoDerm; $n=3$ ) were treated with the indicated concentrations of Melasolv (0.1\%) for 18 days. After treatment, skin equivalents were photographed and the $\triangle \mathrm{L}$ value (de- results obtained using $3 \mathrm{D}$ human skin equivalent suggested that the whitening ability of Melasolv observed in in vitro experiments might also be borne out in clinical trials. Consistent with this possibility, we found that a Melasolv formulation was significantly more effective than vehicle in reducing the appearance of pigmentation $(p<0.05)$ in an 8 -week clinical study conducted on 29 subjects (data not shown), although these findings await further confirmation. Taken together, our findings suggest that Melasolv exerts potent whitening effects.

In the TPEF experiments performed here, we found that a laser power at the sample surface of $>12 \mathrm{~mW}$ activated intrinsic melanin TPEF signals, resulting in artifactually larger and stronger signals. Therefore, it is important to use a lower laser power when comparing melanin TPEF signals in 3D human skin equivalent. Additionally, we confirmed the optimal melanin-detection conditions (excitation, $800 \mathrm{~nm}$; emission, $490-540 \mathrm{~nm}$; laser power, $7.5 \mathrm{~mW}$ ) using a melanocyte-deficient skin tissue model (EpiDermFT, EFT-400; MatTek Corp.), which showed no nonspecific TPEF signals under our experimental conditions.

This report does not provide a mechanism to account for the antimelanogenic effect of Melasolv. However, having demonstrated the whitening efficacy of Melasolv here, we will focus on its action mechanism in the future. In our previous report, we found that Melasolv did not inhibit mushroom tyrosinase activity [5]. Thus, our operating assumption is that the depigmentary action of Melasolv likely reflects its effect on the expression level, and not the activity, of tyrosinase.

Collectively, our results provide an important line of evidence supporting the conclusion that Melasolv has potential for the development of a potent antimelanogenic agent for the treatment of pigmentary disorders and as a cosmetic ingredient for the lightening of darkened skin color.

\section{Acknowledgment}

This research was supported and funded by the Application Technology Research Institute of the Amorepacific R\&D Center. 


\section{Statement of Ethics}

The authors have no ethical conflicts to disclose.

\section{Disclosure Statement}

The authors declare no conflicts of interest.

\section{References}

1 Swalwell H, Latimer J, Haywood RM, BirchMachin MA: Investigating the role of melanin in UVA/UVB- and hydrogen peroxide-induced cellular and mitochondrial ROS production and mitochondrial DNA damage in human melanoma cells. Free Radic Biol Med 2012;52:626-634.

2 Briganti S, Camera E, Picardo M: Chemical and instrumental approaches to treat hyperpigmentation. Pigment Cell Res 2003;16:101110.

3 Ando H, Kondoh H, Ichihashi M, Hearing V: Approaches to identify inhibitors of melanin biosynthesis via the quality control of tyrosinase. J Invest Dermatol 2007;127:751-761.
4 Solano F, Briganti S, Picardo M, Ghanem G: Hypopigmenting agents: an updated review on biological, chemical and clinical aspects. Pigment Cell Res 2006;19:550-571.

5 Kang HH, Rho HS, Hwang JS, Oh SG: Depigmenting activity and low cytotoxicity of alkoxy benzoates or alkoxy cinnamte in cultured melanocytes. Chem Pharm Bull (Tokyo) 2003;51:1085-1088.

6 Lee ES, Park JH, Lee SW, Hahn J, Lee H, Chae SW, Lee TG, Moon DW, Kim SH: Lipid crystals mechanically stimulate adjacent extracellular matrix in advanced atherosclerotic plaques. Atherosclerosis 2014;37:769-776.

7 Han M, Giese G, Schmitz-Valckenberg S, Bindewald-Wittich A, Holz FG, Yu J, Bille JF, Niemz MH: Age-related structural abnormalities in the human retina-choroid complex revealed by two-photon excited autofluorescence imaging. J Biomed Opt 2007;12:024012.
8 Siegrist W, Eberle AN: In situ melanin assay for MSH using mouse B16 melanoma cells in culture. Anal Biochem 1986;159:191-197.

9 Santiago-Walker A, Li L, Haass NK, Herlyn M: Melanocytes: from morphology to application. Skin Pharmacol Physiol 2009;22:114121.

10 Lee CS, Shin YJ, Won C, Lee YS, Park CG, Ye SK, Chung MH: Simvastatin acts as an inhibitor of interferon gamma-induced cycloxygenase-2 expression in human THP-1 cells, but not in murine RAW264.7 cells. Biocell 2009;33:107-114. 